\title{
Tumefactive Demyelinating Lesions: A Case Series of 11 Patients and Review of the Literature
}

\author{
Mustafa Efendioğlu1 , Tuğçe Kızılay², Ruziye Erol Yıldız², (i) Devran Süer², Zerrin Karaaslan³, \\ Cemile Handan Mısırlı2 , (i) Erdem Tüzün ${ }^{3}$, (1) Recai Türkoğlú2
}

${ }^{1}$ Department of Neurosurgery, University of Health Sciences, Hamidiye Faculty of Medicine, Haydarpasa Numune Health Application and Research Center, Istanbul, Turkey

${ }^{2}$ Department of Neurology, University of Health Sciences, Hamidiye Faculty of Medicine, Haydarpasa Numune Health Application and Research Center, Istanbul, Turkey

${ }^{3}$ Department of Neuroscience, Istanbul University, Aziz Sancar Institute of Experimental Medicine, Istanbul, Turkey

\begin{abstract}
Introduction: The aim of this study was to present the follow-up data of 11 patients with tumefactive demyelinating lesions (TDLs) with pointing the challenges of differential diagnosis and indicate the possible prognostic features.

Methods: In our study, a retrospective analysis was performed by collecting clinical, laboratory, and radiological findings with the prospective follow-up of 11 patients with TDL who presented with at least one cerebral demyelinating lesion (>2 cm) and admitted to Haydarpasa Numune Training and Research Hospital between 2000 and 2020.

Results: About $72.7 \%$ of the patients were female $(n=8)$ and $27.2 \%$ were male $(n=3)$. There was a female predominance ( $F /$ $M=2.66$ ) and the mean age of onset was 33.45 years (18-68 years). While TDL was the first neurological event in 9 (81.8\%) of 11 patients, 2 patients (18.1\%) had developed TDL after the diagnosis of multiple sclerosis (MS). Among nine patients whose first neurological event was a tumefactive lesion, three of them were diagnosed with neuromyelitis optica, two patients with MS, and two patients with acute demyelinating encephalomyelitis. Two patients were followed up as TDL with a monophasic course. The average follow-up period was 62.16 months (1-180 months).

Discussion and Conclusion: It is difficult to diagnose TDL by distinguishing it from other lesions, including brain tumors, with clinical and radiological findings. Detailed anamnesis, physical examination, and magnetic resonance imaging can eliminate the need for brain biopsy in patients for the diagnosis. Early diagnosis of TDL and aggressive immunomodulatory treatments may delay the progression to a second demyelinating event or clinically definite MS.

Keywords: Brain tumors; magnetic resonance imaging; multiple sclerosis, tumefactive demyelinating lesion.
\end{abstract}

$\mathrm{T}$ umefactive demyelinating lesion (TDL) is defined as an acute, steroid-responsive, tumor-like inflammatory demyelinating lesion larger than $2 \mathrm{~cm}$, which may have annular enhancement and edema around the lesion with minimal mass effect in the central nervous system ${ }^{[1]}$. This disease spectrum has been reported under various names such as tumor-like demyelinating lesions, TDL, and fulminant multiple sclerosis (MS) lesions ${ }^{[2]}$. TDL is a rare disease with the estimated prevalence of 1-3 cases per 1000 MS cases $^{[3]}$. In the literature, it has been shown that the fe-

Correspondence (iletişim): Tugce Kizilay, M.D. Saglik Bilimleri Universitesi, Hamidiye Tip Fakultesi, Haydarpasa Numune Saglik Uygulama ve Araştırma Merkezi, Noroloji Anabilim Dali, Istanbul, Turkey

Phone (Telefon): +90 5446308688 E-mail (E-posta): drtugcekizilay@gmail.com

Submitted Date (Başvuru Tarihi): 10.05.2021 Accepted Date (Kabul Tarihi): 01.06.2021

Copyright 2021 Haydarpaşa Numune Medical Journal

OPEN ACCESS This is an open access article under the CC BY-NC license (http://creativecommons.org/licenses/by-nc/4.0/).

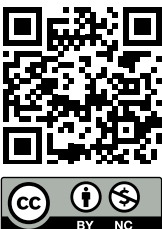


male/male ratio is $3: 1$ with a female preponderance of up to $75 \%$. TDL can occur at any age, with the ages of 20-30 being more frequently affected ${ }^{[4,5]}$.TDL may present as the first neurological event in patients without MS or may occur in patients with a previous diagnosis of $\mathrm{MS}^{[6]}$.

Differing from MS, the clinical presentation often includes headache, cognitive disorders, mental confusion, aphasia, apraxia, hemiparesis, hemisensory deficits, visual field deficits, and/or seizures varying according to lesion's location and size. These atypical clinical and imaging features can mimic brain tumor (glial tumor and primary central nervous system lymphoma), infection, cerebral abscess, and other inflammatory diseases, and may require brain biopsy for differential diagnosis ${ }^{[5]}$.

\section{Materials and Methods}

\section{Subjects}

A retrospective analysis was performed by collecting clinical, laboratory, and radiological findings that were obtained through prospective follow-up of 11 patients with TDL who were admitted to Haydarpasa Numune Training and Research Hospital with at least one cerebral demyelinating lesion $(>2 \mathrm{~cm}$ ) between 2000 and 2020. Patients who were followed up by MS Department with no prior immunosuppressive or immunomodulatory treatment and whose other possible diagnoses that could explain their complaints were ruled out (such as vasculitis, autoimmune diseases, metabolic, and toxic causes) and whose symptoms were relieved after treatment were included in this study. Patients with the history of autoimmune or neoplastic diseases and history of immunosuppressive treatment were excluded from the study.

\section{Statistical Analysis}

Clinical and demographical information were obtained directly from patients or from MS unit patient follow-up files. Patients and their data were reevaluated independently by neurology specialists. The follow-up data of the patients with the neurological findings, course of the disease, treatment types, and pathology results if it was performed were noted.

The scanned radiological parameters in magnetic resonance imaging (MRI) findings were size, localization, and time course of TDLs, any pattern of contrast enhancement, accompanying lesions, mass effect, degree of edema, T1 hypointensity, and the presence of a $\mathrm{T} 2$ hypointense ring. The degree of edema and/or mass effect was graded as follows: (i) Mild: Edema and/or sulcal effacement extending
$<1 \mathrm{~cm}$ from the lesion; (ii) moderate: Edema extending 1-3 $\mathrm{cm}$ from the lesion and/or minimal subfalcine/uncal herniation $<1 \mathrm{~cm}$; and (iii) pronounced: Edema $>3 \mathrm{~cm}$ from the lesion and/or subfalcine/uncal herniation more than $1 \mathrm{~cm}$.

Temporal change of cerebrospinal fluid (CSF) and immunological parameters was evaluated and oligoclonal band profile, serological tests results for certain vasculitis and infectious agents, anti-neuromyelitis optica (NMO) immunoglobulin $\mathrm{G}(\mathrm{lgG})$ antibody serostatus, autoimmune encephalitis, and paraneoplastic panel test results were noted.

\section{Results}

Among all patients with TDL, $72.7 \%$ were female $(n=8)$ and $27.2 \%$ were male $(n=3)$. The average age of onset was 33.45 years (18-68 years), with female predominance $(F / M=2.66)$. Focal TDL was the most common finding $(n=7)$, with frontal, parietal, and temporal lobes being mostly affected. Pons, bulbus, and cerebellum were the other regions that lesions were detected.

While TDL was presented with the first neurological event in 9 of 11 patients (81.8\%), TDL developed after the diagnosis of MS in 2 (18.1\%). Out of the nine patients whose first neurological event was tumefactive lesion, three had a diagnosis of NMO, two had a diagnosis of MS, and two had a diagnosis of acute demyelinating encephalomyelitis (ADEM). The average follow-up period was 62.16 months (1-180 months).

TDLs were resected of the patients number 1,2 , and 3 as a consequence of interpretation of their radiological images as a high-grade glial tumor. However, their pathological findings were found to be compatible with demyelination and they followed up by our MS department.

- Patient number 1: An excisional biopsy was performed due to suspicion of brain tumor. After reevaluation of histopathology with additional immunohistochemical examinations and consultation with reference centers, the patients diagnosed as NMO. Despite steroid and plasmapheresis treatments, the patient developed TDL for 3 times with an interval of 1 year. The patient received rituximab treatment after plasmapheresis and had no attack for the past 4 years

- Patient number 8 had been diagnosed with astrocytoma and received radiotherapy as a result of excisional biopsy performed in an external center after an epileptic seizure 4 years ago. The patients applied to our outpatient clinic with diplopia and right hemi-hypoesthesia, and MRI showed newly added demyelinating lesions in addition to encephalomalasic areas secondary to the 
operation. Frontal mass $(3 \mathrm{~cm} \times 3 \mathrm{~cm})$ that was removed by excisional biopsy was reevaluated and immunohistochemical staining revealed that the pathological changes were compatible with demyelinating disease. Our patient is still being followed up with a diagnosis of secondary progressive MS.

- Patient number 3 was admitted to another hospital 3 months ago for imbalance, nausea, and vomiting, and an excisional biopsy from the cerebellar hemisphere was made for diagnostic purpose. Pathological changes were suggested encephalomyelitis with demyelination. When the patient referred to our clinic, she diagnosed with ADEM considering clinical and radiological findings. Despite intensive treatment with intravenous methyl prednisolone (IVMP), plasmapheresis, cyclophosphamide, and anti-edematous drugs, the disease rapidly progressed and she died

- Patient number 5 had a tumefactive brainstem lesion that was interpreted as brainstem encephalitis. There was a vaccination story as a trigger factor 1 month before onset of the symptoms. After 10 days of IVMP and oral steroid treatment, his hemiparesis with $1 / 5$ muscle strength and incontinence recovered in 6 months

- Patient number 2 presented as isolated TDL but in his follow-up MRI, new lesions were detected in the left mesencephalon, pons, and cervical spine at C2-C3 vertebra level. Teriflunomide treatment was started on and the patient had no further attack under treatment

- Patients number 4 and 6 showed monophasic TDL course and after IVMP their complaints completely resolved. In their follow-up MR after 1 year, MRI lesions were seemed to be decreased in size

- Patients number 9 and 11 were following with MS and TDL was added on through disease course. Patient number 11 developed a TDL during a steroid-resistant MS attack under fingolimod treatment. He recovered after plasmapheresis and natalizumab treatment was started

- Patients number 1, 7, and 10 were diagnosed with NMO spectrum disease after TDLs and they received IVMP and plasmapheresis as attack treatment.

Detailed clinical, radiological, and immunological findings of the patients are presented in Table 1.

\section{Discussion}

TDL is a rare disease of the central nervous system. It is often present in the patients with a history of MS as a first demyelinating attack as well as in other primary demyelinating diseases such as ADEM or seropositive $\mathrm{NMO}^{[2]}$. The immunopathogenesis of TDL is not fully elucidated yet. Previous articles have reported that TDL develops after vaccination, and TDL was originally thought to be an intermediate form between MS and ADEM. However, subsequent studies could not confirm that kind of relationship ${ }^{[7,8]}$. The presence of oligoclonal bands (OCBs) in the CSF of patients with both TDL and MS, and case series of TDL patients that reported good response to plasma exchange and rituximab treatments indicate a common etiology for antibody-mediated and B-cell-mediated immunological mechanisms for both diseases ${ }^{[1]}$. In some reported cases, such as in patient number 11 in our study, tumefactive lesions develop during fingolimod treatment, suggesting a causal role for fingolimod, but the mechanism is not fully understood. The possible mechanism explaining fingolimod relationship with TDL may be a paradoxical effect of fingolimod, which affects inhibitory immune cells and causes lymphocyte subset shift with increased disease activity and large demyelinating lesions ${ }^{[9]}$.

Histopathology can be misinterpreted in up to $31 \%$ of cases. In the biopsy materials, findings that were suggesting of include low-grade astrocytoma (the most common), high-grade astrocytoma, oligodendroglioma, and other non-neoplastic lesions could be observed, given the hypercellular nature of these lesions and the frequent presence of atypical reactive astrocytes (i.e., Creutzfeldt-Peters cells) and mitotic figures. This misdiagnosis can lead to inflammatory demyelinating patients receiving radiotherapy, which is known to exacerbate the disease $\mathrm{e}^{[5]}$. Our patient number 8 was also diagnosed with low-grade glioma and received RT in, however, when the biopsy materials of the patient were reevaluated, it was found to be compatible with demyelinating disease. CSF examination was repeated and OCBs were detected.

Additional tests may be required to rule out other pathologies such as vasculitis, granuloma, infection, and malignancy. These tests include serology, infection, vasculitis and malignancy screening tests, and CSF analysis $^{[10]}$. The main findings in CSF analysis are the increase in IgG index and OCBs. The result of unpaired positive OCBs in the CSF may be present in up to $90 \%$ of patients previously diagnosed with MS, compared to $52 \%$ of patients presenting with tumefactive demyelination as the first clinical event ${ }^{[1,6]}$. In our study, OCBs were detected in the CSF in three patients, while NMO antibodies were detected in two patients. 

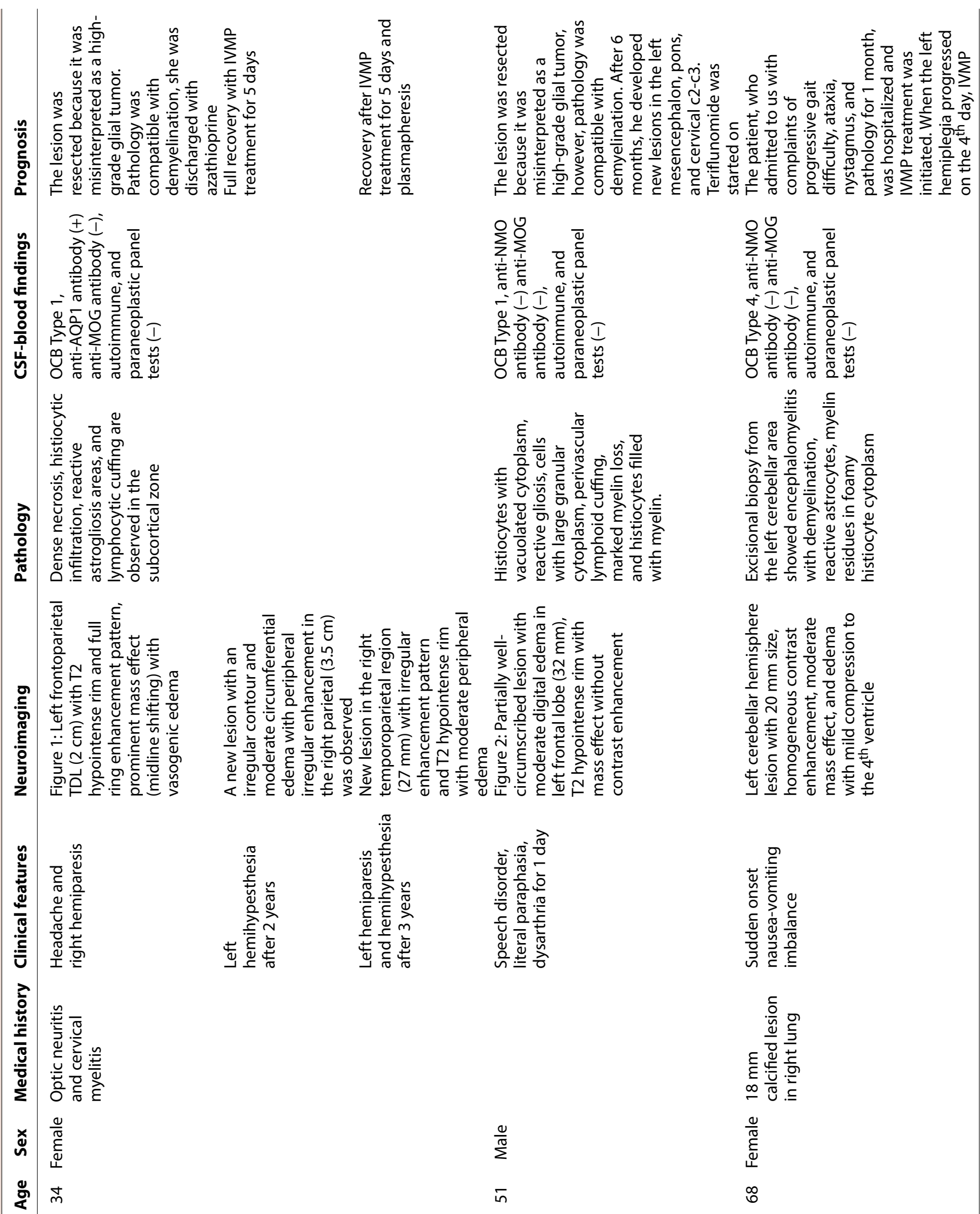


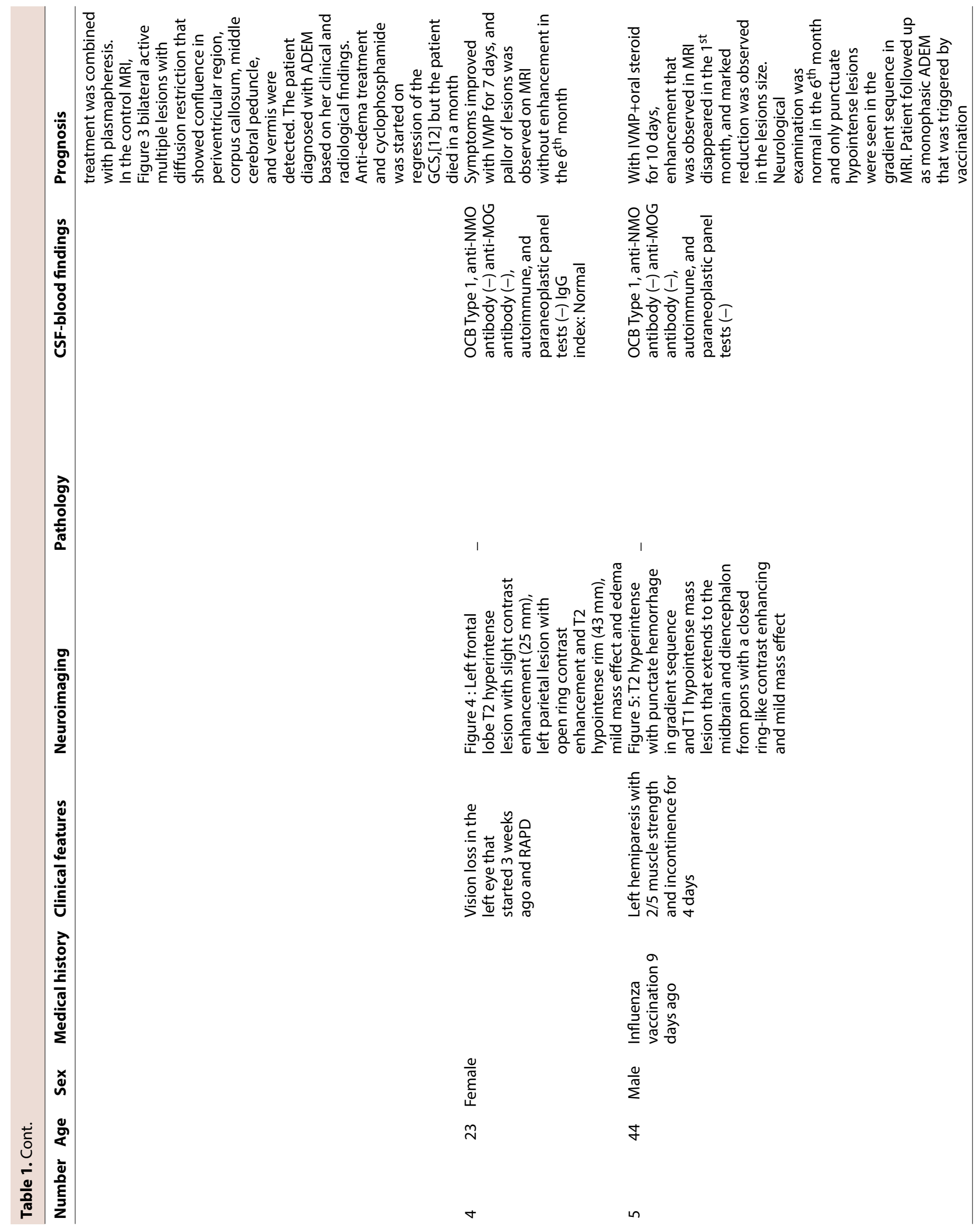




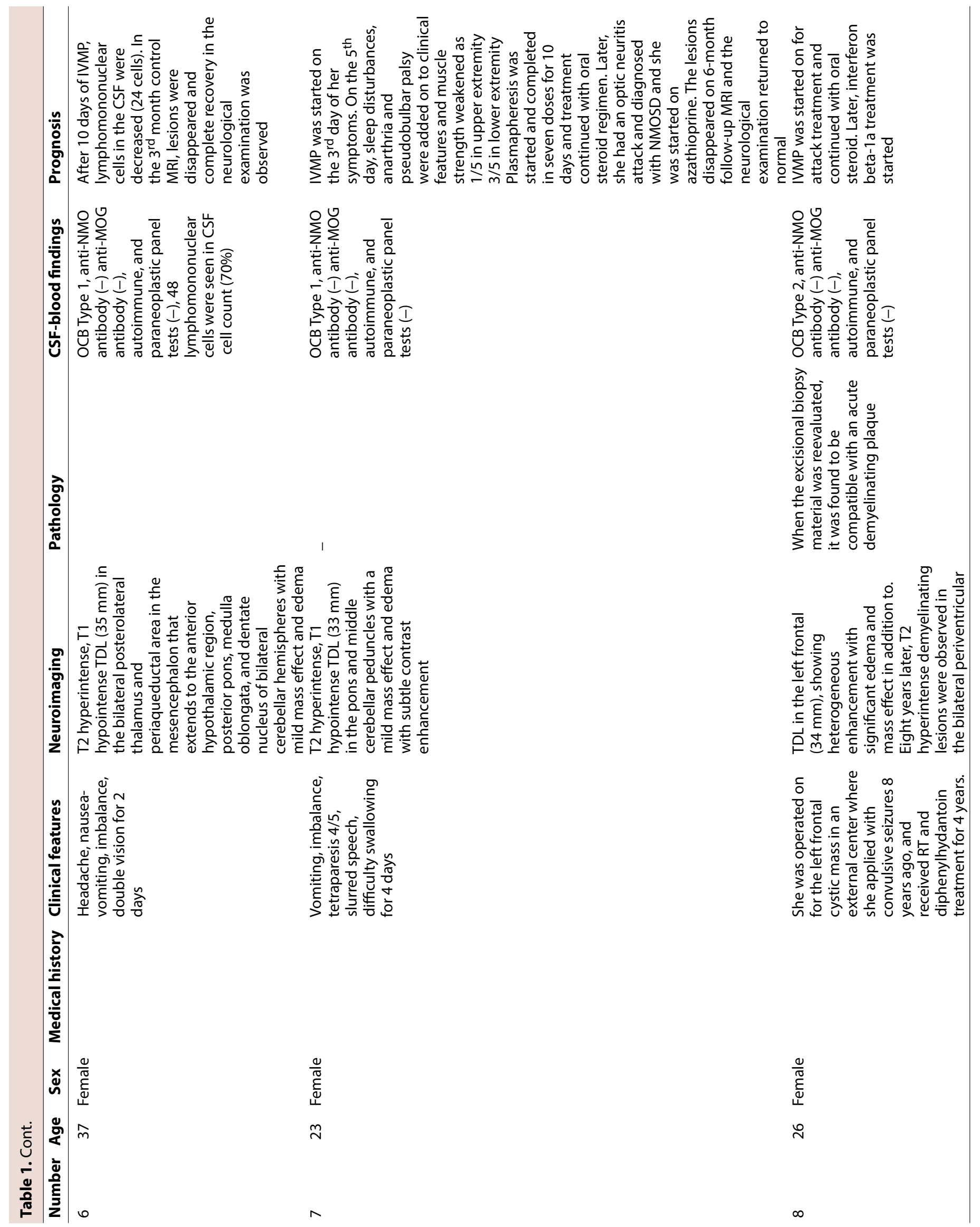




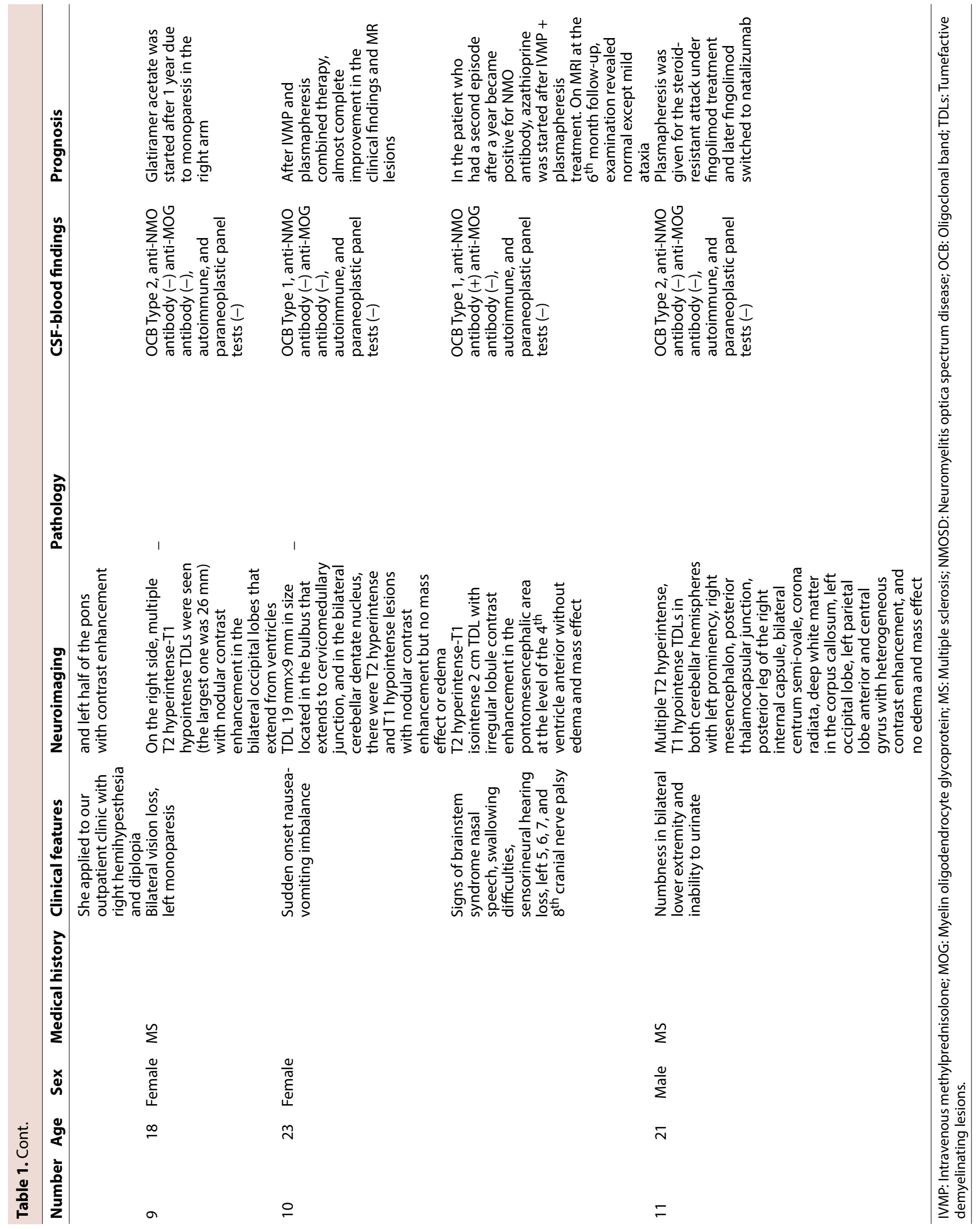




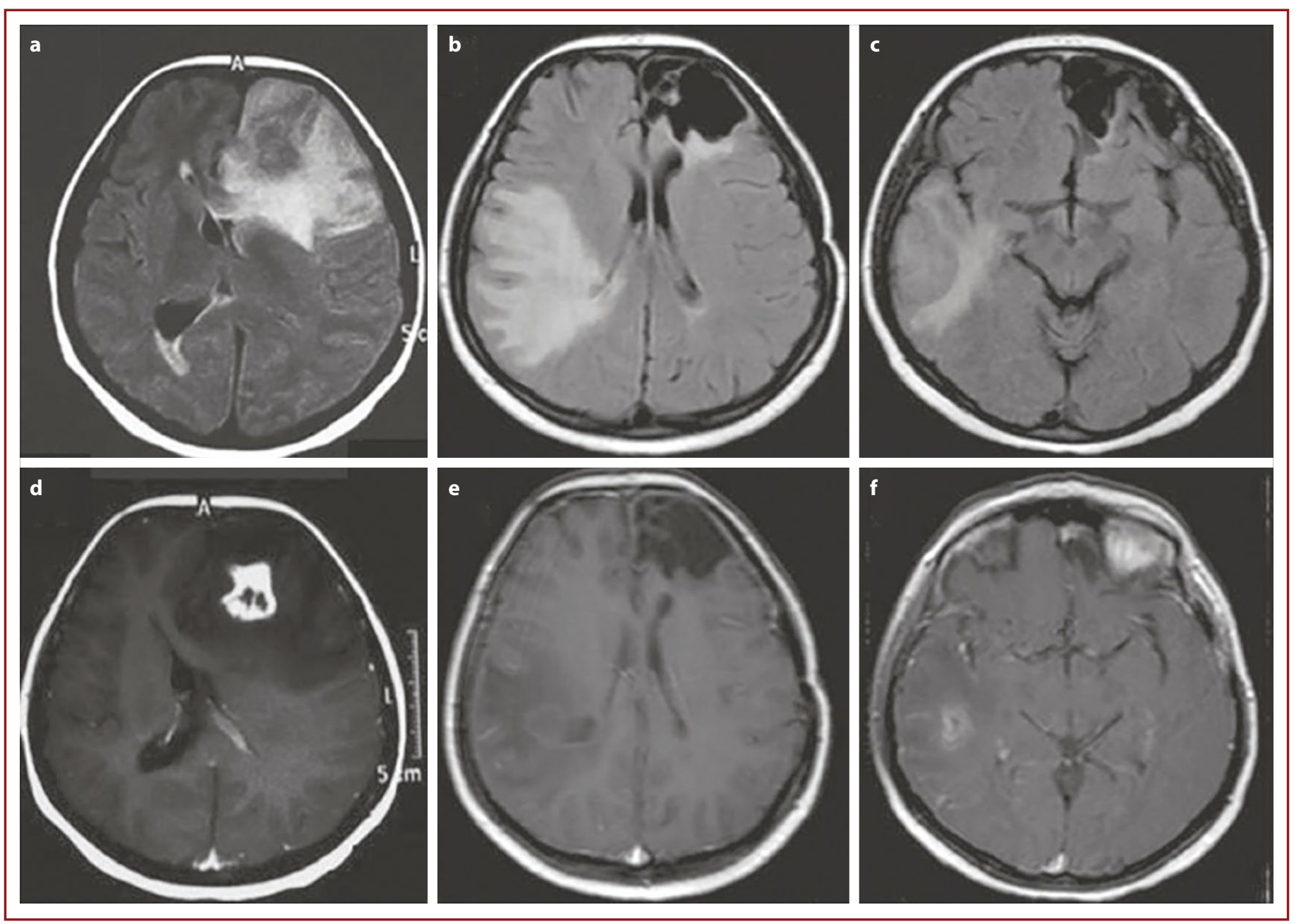

Figure 1. Shows MRI of patient. 1. Axial FLAIR (a-c) and gadolinium-enhanced T1-weighted (d-f) cranial MRI images obtained during consecutive tumefactive demyelination episodes. MRI image obtained during the first episode $(\mathbf{a}, \mathbf{d})$ reveals an extensive brain lesion in the left frontoparietal region causing midline shift. T1-weighted axial cranial MRI image with contrast enhancement (d) shows a ring-enhancing lesion. In second $(\mathbf{b}, \mathbf{e})$ and third $(\mathbf{c}, \mathbf{f})$ episodes, FLAIR $(\mathbf{b}, \mathbf{c})$ and gadolinium-enhanced T1-weighted $(\mathbf{e}, \mathbf{f})$ images show hyperintense extensive brain lesions in the right temporoparietal region with moderate contrast enhancement.

There are several important radiological features that indicate demyelination. Tumefactive lesions typically affect white matter, but gray matter may also be affected. Most TDL are focal and supratentorial with a tendency toward the frontal and parietal lobes. However, other areas of the cerebral hemispheres such as deep gray matter, brainstem, cerebellum, and medulla spinalis are less affected. TDLs were seen as well-circumscribed, hypodense areas in computed tomography, while on MRI, they are hyperintense in T2 and hypointense in T1. Contrast enhancement of TDL lesions is seen in $95-100 \%$ of patients with variable patterns including open or closed rings, diffuse, homogeneous, dotted, or concentric patterns. The open ring enhancement pattern used in the differential diagnosis of solitary lesions from neoplasms. The open ring enhancement pattern with open part of the ring showing the gray matter or deep nu- clei of the cortex suggests a demyelinating etiology ${ }^{[11]}$. Lesions have mass effect in approximately of $45 \%$ of patients and associated with edema in approximately $77 \%$ of the patients ${ }^{[5]}$. Other less common abnormalities include a peripheral restriction on diffusion-weighted images and venous enhancement or dilatation surrounding the lesion on brain angiography ${ }^{[12]}$. The T2 hypointense rim is often found in the same ring enhancement area, and in most of the lesions, there is associated T1 hypointensity for some degree. Other useful radiological findings, although nonspecific, are the relative absence of mass effect and edema, given the size of the lesions ${ }^{[13]}$.

In MR spectroscopy (MRS) neuroimaging technique, TDLs may cause an increase in choline: $\mathrm{N}$-acetylaspartate ratio, which is also common in neoplasms. Another finding that supports TDL diagnosis is the increased glutamate/glu- 


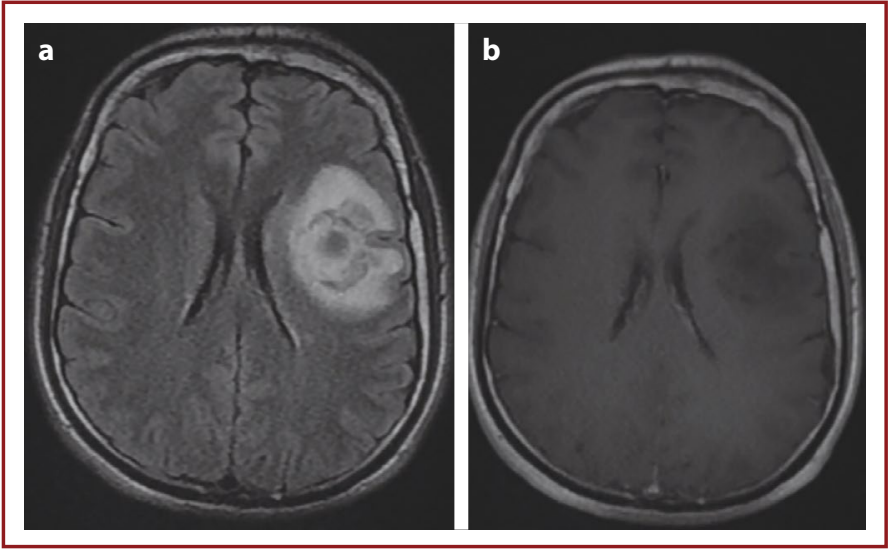

Figure 2. (Patient 2): (a) Partially well circumscribed lesion with moderate digital edema in left frontal lobe $(32 \mathrm{~mm})$, (b) T2 hypointense rim with mass effect without contrast enhancement.

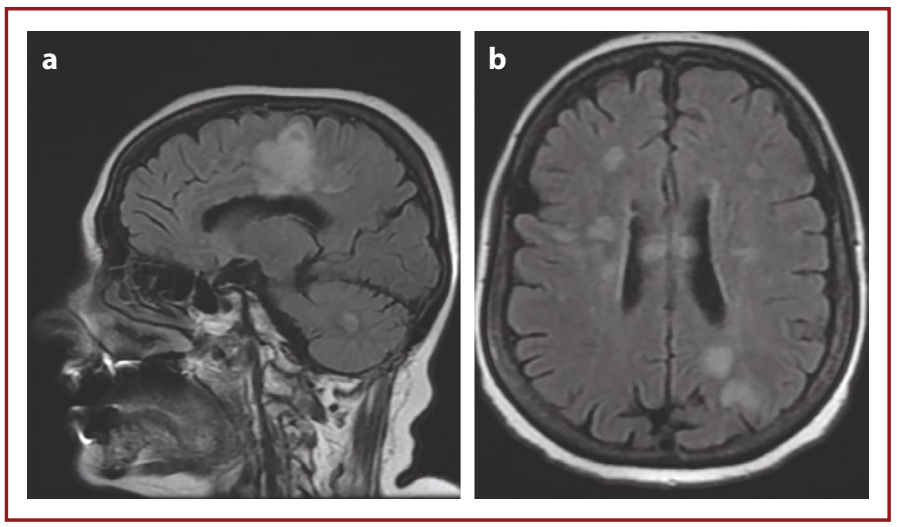

Figure 3. (Patient 3): (a, b) Bilateral active multiple lesions that showed confluence in periventricular region, corpus callosum, middle cerebral peduncle and vermis were detected. The patient diagnosed with ADEM.

tamine peak. Serial MRS is more beneficial than a single imaging because TDLs tend to show variations in different imaging, while neoplasms tend to show consistent signs of progressive disease activity ${ }^{[14]}$.

The treatment approach is mostly based on the personal experience of physicians in the light of literatüre knowledge. Corticosteroids are considered first-line therapy for acute symptomatic TDLs, and more than $80 \%$ of patients recover after steroid treatment ${ }^{[1,6]}$. Plasmapheresis is considered second-line therapy for patients who do not respond to corticosteroids or as an adjunct therapy in severe cases $^{[15-17]}$. Rituximab, an anti-CD20 B-cell-monoclonal antibody, has been reported in some case reports as the treatment of acute fulminant cases that do not respond to both corticosteroids and plasmapheresis ${ }^{[18]}$. Cyclophosphamide and intravenous IG may exhibit some beneficial effects, more commonly in pediatric patients ${ }^{[19]}$. There is not enough evidence to support MS disease-modifying

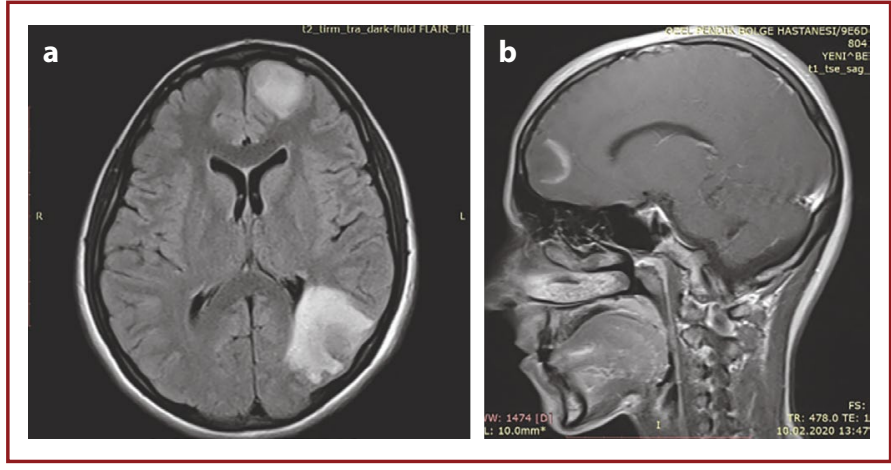

Figure 4. (Patient 4): (a) Left frontal lobe T2 hyperintense lesion with slight contrast enhancement $(25 \mathrm{~mm})$, (b) Left parietal lesion with open ring contrast enhancement and $\mathrm{T} 2$ hypointense rim $(43 \mathrm{~mm})$, mild mass effect and oedema.

treatment in the patients with first episode tumefactive lesions, if the dissemination in space and time criteria of MS could not be met with clinical or paraclinical findings. Most clinicians prefer to use disease-modifying therapy if the patient meets diagnostic criteria for $\mathrm{MS}^{[4]}$. The presence of parameters that increase the risk of conversion to MS, such as considering the TDL attacks as equivalent to the clinical isolated syndrome, a severe and disabling attack or detection of OCBs in the CSF could be consider among the supportive arguments that suggest initiation of treatment,. It is well known that initiating disease-modifying therapy after clinically isolated syndrome delays a second attack and thus the conversion to MS ${ }^{[6]}$. CSF findings suggesting of MS were added to revised McDonald's criteria in 2017. Therefore, we started teriflunomide, one of the MS modifying therapies, in patient number 2 , which is a clinically isolated syndrome that does not fulfill the MS diagnostic criteria.

The long-term prognosis depends on whether the patient has an established diagnosis of MS or if TDL appears as the first clinical event. The prognosis is well if disease detected early and treated appropriately. Approximately 6-70\% of patients presenting with TDL as their first clinical event develop clinically definite $\mathrm{MS}^{[1]}$. This is a wide range and it is accepted in the literature that approximately two-thirds of patients will follow a relapsing-remitting course. These patients have a similar long-term disability to other MS patients. It has been shown that $1 / 3$ of the patients do not develop demyelinating attacks in the future ${ }^{[9]}$.

\section{Conclusion}

It is difficult to diagnose TDL by distinguishing it from other lesions, including brain tumor, with clinical and radiological findings. Biopsies performed with a brain tumor suspicion can reveal findings similar to a brain tumor such as 


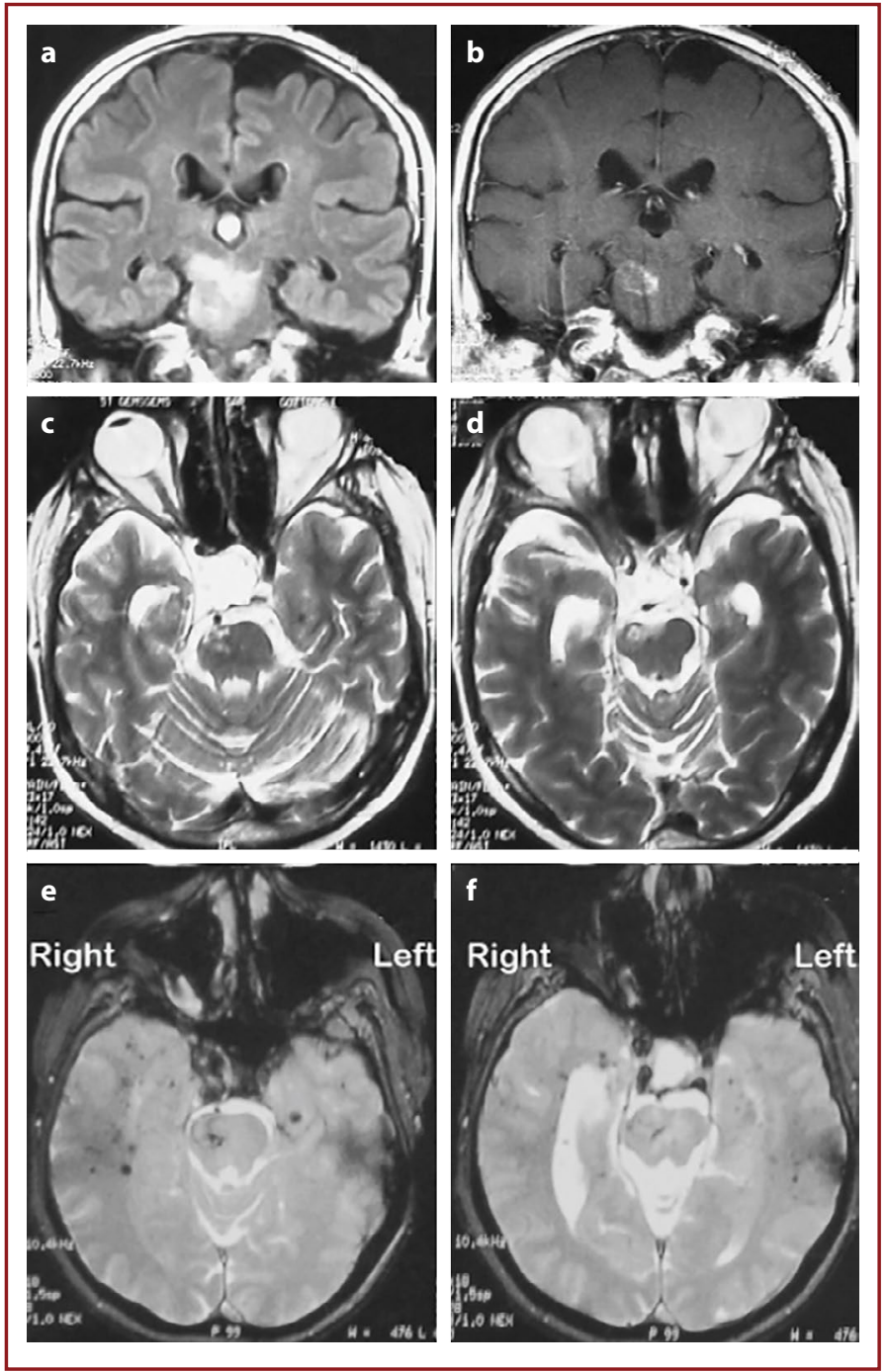

Figure 5. (Patient 5): A brainstem encephalitis following influenza vaccination FLAIR-weighted coronal section of brain MRI shows a massive hyperintense lesion in the brainstem extending to the diencephalon (a) and enhancing contrast on coronal T1-weighted section (b). One month later, T2-weighted axial images still show the lesion in pontine (c) and midbrain (d) sections. Corresponding gradient axial images $(\mathbf{e}, \mathbf{f})$ show foci of hypointensities suggesting microhaemorrhages not only on the lesion site in the brainstem but also in both cerebral hemispheres.

lymphomas. Steroid treatment may increase the risk of misdiagnosis of the central nervous system lymphoma since it will reduce the contrast enhancement and brain edema.

Detailed history, physical examination, and serial MRI imaging can eliminate the need for a brain biopsy in patients. Early diagnosis and aggressive immunomodulatory treatments may delay the progression to a second demyelinating event or clinically definite MS. It can be predicted that follow-up of the patients with this clinical features by the physicians and centers specialized in MS will reduce the possibility of misdiagnosis and unnecessary operations.
Ethics Committee Approval: Study was approved by the Ethics Committee of University of Health Sciences Hamidiye Faculty of Medicine (date: 21/01/2016, number: 2015/KK/96).

Peer-review: Externally peer-reviewed.

Authorship Contributions: Concept: M.E.; Design: R.T.; Supervision: E.T.; Funding: None; Materials: R.E.Y.; Data Collection or Processing: T.K.; Analysis or Interpretation: Z.K.; Literature Search: D.S.; Writing:T.K.; Critical Review: C.H.M.

\section{Conflict of Interest: None declared.}

Financial Disclosure: The authors declared that this study received no financial support.

\section{References}

1. Hardy TA, Chataway J. Tumefactive demyelination: An approach to diagnosis and management. J Neurol Neurosurg Psychiatry 2013;84:1047-53. [CrossRef]

2. Xıa L, Lin S, Wang ZC, Li SW, Xu L, Wu J, et al. Tumefactive demyelinating lesions: Nine cases and a review of the literature. Neurosurg Rev 2009;32:171-9. [CrossRef]

3. Frederıck MC, Cameron MH. Tumefactive demyelinating lesions in multiple sclerosis and associated disorders. Curr Neurol Neurosci Rep 2016;16:26. [CrossRef]

4. Comı G. Multiple sclerosis: Pseudotumoral forms. Neurol Sci 2004;25:s374-9. [CrossRef]

5. Lucchınettı CF, Gavrilova RH, Metz I, Parisi JE, Scheithauer BW, Weigand S, et al. Clinical and radiographic spectrum of pathologically confirmed tumefactive multiple sclerosis. Brain 2008;131:1759-75. [CrossRef]

6. Altıntas A, Petek B, Isik N, Terzi M, Bolukbasi F, Tavsanli M, et al. Clinical and radiological characteristics of tumefactive demyelinating lesions: Follow-up study. Mult Scler 2012;18:1448-53. [CrossRef]

7. Kepes JJ. Large focal tumor-like demyelinating lesions of the brain: Intermediate entity between multiple sclerosis and acute disseminated encephalomyelitis? A study of 31 patients. Ann Neurol 1993;33:18-27. [CrossRef]

8. Wattamwar PR, et al. Evolution and long term outcome in patients presenting with large demyelinating lesions as their first clinical event. J Neurol Sci 2010;297:29-35.[CrossRef]

9. Algahtanı $H$, Shırah B, Alassırı A. Tumefactive demyelinating lesions: A comprehensive review. Mult Scler Relat Disord 2017;14:72-9. [CrossRef]

10. Scott BJ, Douglas VC, Tihan T, Rubenstein JL, Josephson SA. A systematic approach to the diagnosis of suspected central nervous system lymphoma. JAMA Neurol 2013;70:311-9. [CrossRef]

11. de Medeıros FC, de Albuquerque LA, Pittella JE, de Souza RB, Neto AP, Christo PP. Open-ring enhancement in pseudotumoral multiple sclerosis: Important radiological aspect. Case Rep Neurol Med 2014;2014:951690. [CrossRef]

12. Kırıyama T, Kataoka H, Taoka T, Tonomura $Y$, Terashima M, Morikawa $M$, et al. Characteristic neuroimaging in patients with tumefactive demyelinating lesions exceeding $30 \mathrm{~mm}$. J Neuroimaging 2011;21:e69-77. [CrossRef] 
13. Dagher AP, Smırnıtopoulos J. Tumefactive demyelinating lesions. Neuroradiology 1996;38:560-5. [CrossRef]

14. Butterıss DJ, Ismail A, Ellison DW, Birchall D. Use of serial proton magnetic resonance spectroscopy to differentiate low grade glioma from tumefactive plaque in a patient with multiple sclerosis. Br J Radiol 2003;76:662-5. [CrossRef]

15. Selfert CL, Wegner C, Sprenger T, Weber MS, Brück W, Hemmer $B$, et al. Favourable response to plasma exchange in tumefactive CNS demyelination with delayed B-cell response. Mult Scler J 2012;18:1045-9. [CrossRef]

16. Paus S, Braff S, Pendlebury W, Panitch $\mathrm{H}$. Treatment of steroidunresponsive tumefactive demyelinating disease with plasma exchange. Neurology 2003;61:1022-3. [CrossRef]

17. Weınshenker BG, O'Brien PC, Petterson TM, Noseworthy JH, Lucchinetti CF, Dodick DW, et al. A randomized trial of plasma exchange in acute central nervous system inflammatory demyelinating disease. Ann Neurol 1999;46:878-86. [CrossRef]

18. Fan X, Mahta A, De Jager PL, Kesari S. Rituximab for tumefactive inflammatory demyelination: A case report. Clin Neurol Neurosurg 2012;114:1326-8. [CrossRef]

19. Dastgır J. Dımarıo JR, Francis J. Acute tumefactive demyelinating lesions in a pediatric patient with known diagnosis of multiple sclerosis: Review of the literature and treatment proposal. J Child Neurol 2009;24:431-7. [CrossRef] 